\title{
Fiducia: male oscuro ma curabile del SSN
}

\author{
di Elio Borgonovi
}

Si parla tanto di tutela della salute e forse poco di salute del SSN. A dire il vero si parla della crisi reale, temuta, prevista, preannunciata, del SSN ma forse in termini riduttivi. Per affrontare il problema occorre partire da una triplice contraddizione. In primo luogo, si può dire che con il passare degli anni è migliorata notevolmente la capacità di tutela della salute delle persone, mentre è forse diminuita la capacità di tutelare la salute del SSN, intesa come livello di funzionalità. In secondo luogo, per quanto riguarda la salute delle persone si è passati dal concetto di "lotta alla malattia" a quello di recupero, mantenimento, promozione del benessere fisico, psichico, relazionale, sociale, spirituale (in senso laico o religioso) delle persone, mentre si può dire che per quanto riguarda il SSN e le ASL e AO che le compongono si è ancora nella fase di "lotta alla malattia" intesa come lotta all' inappropriatezza, agli sprechi, alle inefficienze. In terzo luogo, per quanto riguarda la condizione di salute delle persone si è affermato il concetto di multidimensionalità in quanto si parla di comorbilità e multicronicità, mentre per quanto riguarda l'analisi sul servizio sanitario nazionale e sulle ASL e AO le analisi sono prevalentemente mono-dimensionali in quanto si sottolinea come causa la mancanza di finanziamenti adeguati, la carenza di personale, il ritardo rispetto all'evoluzione delle conoscenze.

Per cercare di superare questi limiti e per rimanere nell'analogia con i processi di tutela della salute si propone una revisione/integrazione della diagnosi. Infatti, solo cambiando la diagnosi si possono cambiare anche le terapie per aumentare l'efficacia delle stesse. Si può dire che le difficoltà dei sistemi di tutela della salute, non solo quello italiano, sono influenzate, oltre che dai fattori comunemente evidenziati (allungamento della vita e conseguente invecchiamento della popolazione, aumento delle opportunità consentite dal progresso scientifico rispetto alla carenza di risorse, impatto delle nuove tecnologie), anche da due ordini di motivi di carattere generale tra loro strettamente connessi: la crisi dei sistemi di rappresentanza e la crisi di fiducia. Nella fase della medicina pre-scientifica la condizione di salute era collegata alla concezione religiosa sia per quanto riguarda la condizione dei pazienti sia per quanto riguarda molti di coloro che si dedicavano all'assistenza (monaci, suore, persone dotate di una vocazione). Nella fase scientifica la tutela della salute è stata collegata alle conoscenze, al concetto di evidenze scientifiche, sintetizzate nel principio della evidence-based medicine. L'era della razionalità ha contribuito a staccare il problema della salute da quello della fede. Questa fase si è sviluppata nel mondo occidentale contestualmente all'affermarsi dei sistemi di rappresentanza nei quali vi era un equilibrio tra fiducia nelle conoscenze e fiducia nei sistemi di rappresentanza.

Soprattutto nel nuovo secolo la crisi dei sistemi di rappresentanza (politica, istituzionale, sociale) e la crisi di fiducia nella società della razionalità si sono alimentate a vicenda in un circuito che sembra aver assunto un'accelerazione progressiva, anche se alcuni sintomi si erano manifestati già negli anni Novanta del secolo scorso. Da alcuni decenni il mondo occidentale si è trovato in una condizione che in termini medici avrebbe previsto la prevenzione secondaria o diagnosi precoce e che in termini manageriali e di policy-making si può definire come "segnali deboli" da affrontare tempestivamente. Non essendo intervenuti, oggi questo mondo si trova in una fase acuta della malattia, che si cerca di affrontare con rimedi tradizionali ma che sembrano poco efficaci. La perdita di fiducia nei sistemi di democrazia rappresentativa è stata determinata dall'indebolimento dei "corpi intermedi della società". Il positivismo scientifico 
aveva sostituito la fede religiosa con la fiducia nella razionalità, resa credibile e accettata in relazione al ruolo di corpi intermedi: la comunità scientifica, l'impresa e il mercato per la razionalità economica; il bilanciamento dei poteri nello Stato moderno per la razionalità istituzionale, l'equilibrio tra varie forme di aggregazione e rappresentanze sociali. L'indebolimento dei corpi intermedi, che ha portato alla società liquida, tema centrale delle analisi del sociologo Zygmunt Bauman, e alla cultura dell'eccellenza individualistica, ha determinato una crisi di fiducia i cui riflessi sono rilevanti nel sistema di tutela della salute.

La fiducia è un fattore determinante per i comportamenti, tale rapporto può essere sintetizzato nei seguenti termini: fiducia $\rightarrow$ credibilità $\rightarrow$ comportamenti (nel caso specifico dei pazienti). Nonostante i molti progressi della scienza si può dire che questo rapporto si sia rotto in molti pazienti per vari motivi. Innanzitutto perché i medici e gli altri professionisti si sono posti in un atteggiamento di "superiorità" nei confronti del paziente in quanto detentori delle conoscenze idonee a definire il bisogno (diagnosi) e individuare la risposta (terapia): una situazione di asimmetria accentuata anche dal fatto che il paziente si trova in una situazione di debolezza anche sul piano emotivo per ciò che riguarda il proprio stato di salute. Una condizione che lo porta ad affidarsi al medico, all'infermiere o ad altro professionista della salute. Con l'aumento del livello di istruzione della popolazione e, negli ultimi decenni, con la diffusione delle informazioni scientifiche anche in Rete è stata superata questa asimmetria in termini positivi o negativi. Positivi, poiché il paziente è in grado di accedere in tempo reale e senza costi a informazioni riguardanti la salute, con un'accessibilità molto più rapida e diversa da quella garantita dal consulto di più medici come accadeva in passato. In senso negativo, in quanto una gran parte delle decine di migliaia di siti che riguardano la salute dà informazioni volutamente distorte, perché per esempio finalizzate a promuovere il consumo di farmaci, integratori o altri prodotti, non complete e comunque non interpretabili correttamente da chi non ha conoscenze di base di medicina. In molti casi di fronte al manifestarsi di certi sintomi i pazienti per prima cosa cercano informazioni in Rete o anche tramite $i$ gruppi social e poi vanno dal medico per avere conferma dell'auto-diagnosi o addirittura per ottenere formalmente le prescrizioni. In moltissimi altri casi dopo la diagnosi e la terapia suggerita dal medico i pazienti accedono alla Rete o si confrontano nei gruppi social per avere una conferma di ciò che ha detto il medico. L'accesso a queste informazioni ha messo in crisi molti dei rapporti di fiducia con il singolo medico, infermiere o altro professionista della salute $e$ quella forma di "sacralità" che per decenni aveva caratterizzato queste professioni, minandone la credibilità.

Un secondo motivo che ha rotto la catena fiducia $\rightarrow$ credibilità $\rightarrow$ comportamenti è intrinseco allo sviluppo delle conoscenze. Esso è talmente rapido da rendere impossibile per il medico, di medicina generale o anche specialista, tenersi aggiornato. Indubbiamente anche medici, infermieri e altri professionisti della salute hanno a disposizione strumenti per accedere in tempo reale alle conoscenze e alle informazioni, ma non va trascurato il fatto che ogni medico, infermiere o altro professionista della salute interagisce con pazienti molto diversi, mentre il singolo paziente può concentrare la raccolta di informazioni con riferimento alla propria specificità. Anche quando appartengono a gruppi formalmente omogenei, per esempio cardiopatici, neurologici, oncologici, o a sottogruppi, per esempio diversi tipi di cardiopatie, patologie neurologiche o tumori, i pazienti sono diversi e si aspettano risposte al proprio bisogno specifico. I medici, gli infermieri e gli altri professionisti della salute che hanno una cultura di "personalizzazione del rapporto" riescono a mantenere la fiducia perché ascoltano i pazienti, danno informazioni comprensibili, fanno comprendere quali possono essere i limiti delle conoscenze mediche. Non si presentano come i detentori di una conoscenza "scientifica e non discutibile" ma coinvolgono il paziente nel ricercare insieme le soluzioni che meglio si adattano allo stesso. Non solo per atteggiamento personale, ma anche a causa di pressioni determinate dalla ricerca di efficienza di unità organizzative, ospedali e ASL, dalla scarsità di risorse o di attrezzature e da altri condizionamenti esterni, sembra ridursi il numero di medici, infermieri e altri professionisti della salute che riescono a mantenere questo rapporto di fiducia. Senza voler dare una valutazione sul piano quantitativo relativamente ai professionisti della salute in grado di suscitare e mantenere un rapporto di fiducia e a quelli nei confronti dei quali la fiducia viene meno, si può dire che nella società dell' informazione purtroppo si diffondono più rapidamente le informazioni di tipo negativo rispetto a quelle di tipo positivo. Il paziente che ha riscontrato comportamenti "non professionali" tende a segnalarlo in Rete e queste segnalazioni si diffondono e rischiano addirittura di diventare virali, incidendo sulla credibilità non solo del 
singolo professionista ma dell'intera professione, se non addirittura del SSN. Questa situazione può essere affrontata sottolineando che oltre a far bene (risposte appropriate ed efficaci ai bisogni di salute) e a far bene il bene (garantire servizi efficienti e senza lunghe liste di attesa), oggi la sfida delle professioni sanitarie, delle aziende e del SSN è quella di far conoscere bene, il bene che si fa bene. Per esempio, con siti certificati che danno informazioni corrette, diffusione di informazioni positive che possono bilanciare informazioni o percezioni negative.

Un terzo motivo che mina il rapporto fiducia $\rightarrow$ credibilità $\rightarrow$ comportamenti è collegato alla divaricazione che esiste tra realtà, percezione e aspettative. Servizi di tutela della salute svolti in modo appropriato, efficace ed efficiente possono essere percepiti come inadeguati se non sono comunicati in modo corretto o comprensibile dai pazienti. Inoltre, in una società nella quale vi è giustamente una forte attenzione ai diritti dei pazienti oltre che ad altri diritti umani e della persona, si generano aspettative molto elevate che aumentano la divaricazione tra qualità reale delle risposte ai bisogni di salute e percezione da parte dei pazienti. Lo sviluppo delle conoscenze, da un lato, e i sistemi di tutela giuridica dei diritti, dall'altro, costituiscono un tensore positivo in quanto aumentano le aspettative e stimolano il sistema di offerta al continuo miglioramento. Tuttavia, se non sono adeguatamente governati portano a una situazione di divaricazione e a squilibri che minano il rapporto di fiducia e che determinano comportamenti dei pazienti che si riflettono negativamente sul funzionamento dell' intero sistema.

La perdita di fiducia genera un "paradosso" tipico delle società moderne di cui si sottolineano solo alcune dimensioni. L'aumento delle aspettative di tanti gruppi di pazienti alimentati dalla consapevolezza che è possibile affrontare le proprie esigenze genera pressioni nei confronti di coloro che devono definire le priorità, data la limitatezza delle risorse. Coloro che devono compiere scelte politiche in senso stretto o di "politica di allocazione delle risorse destinate alla tutela della tutela" deludono un numero crescente di pazienti anche quando compiono scelte "razionali" e in tempi adeguati. I delusi si fanno sentire in modo molto più efficace di quanto non accada a coloro che beneficiano delle decisioni. Ad aggravare questo meccanismo concorrono scelte politiche e di politica sanitaria non razionali con riguardo al rapporto benefici/costi, caratterizzate da compromessi anche sul piano tecnico, giuridico e organizzativo, in tempi lunghi. Un'altra dimensione del paradosso è legata al seguente meccanismo. La presenza di riconoscimento formale di diritti può indurre coloro che si ritengono penalizzati da certe decisioni a promuovere ricorsi sul piano giuridico, annullare decisioni, bloccare l'utilizzo di risorse o l'avvio di servizi, determinando effetti negativi sulle aspettative di altri pazienti. È il tipico paradosso di contrasto di interessi che invece di generare comportamenti a beneficio di molti (win-win) causa effetti negativi per tutti (lose-lose). Una terza dimensione del paradosso deriva dal principio di trasparenza, che in sé è positivo e deve essere perseguito, ma che se utilizzato in senso improprio determina conseguen$z e$ "non volute di tipo negativo". Soprattutto con riferimento alle aziende e ai servizi pubblici, il principio di trasparenza non di rado impone obblighi che si traducono in appesantimenti di tipo burocratico-formale, ricorsi contro presunti comportamenti in contrasto con normative, interventi dell'autorità giudiziaria che sequestra documenti, blocca attività, con riflessi negativi sulla funzionalità delle aziende sanitarie e ospedaliere. Si ripete che il principio di trasparenza deve guidare il comportamento di chi utilizza risorse pubbliche acquisite tramite la fiscalità generale, come è il caso del SSN, ma l'abuso o l'applicazione inutilmente formale di tale principio non di rado interferisce con la funzionalità e produce effetti negativi sui pazienti. Una quarta e ultima dimensione del paradosso riguarda l'evoluzione delle professionalità. La tutela della salute richiede professionalità nuove mentre le regole della selezione e assunzione nel settore pubblico impediscono o comunque rendono difficile l'inserimento di figure nuove. Si tratta di un circolo vizioso che penalizza soprattutto le aziende pubbliche e che nell'ultimo decennio in Italia è stato ancor più accentuato dai vincoli quantitativi riguardanti il personale.

Il tema della comorbilità legato alla fiducia non riguarda solo il rapporto tra medici, infermieri e altri professionisti della salute ma si estende anche al rapporto tra professionisti della salute e manager, in quanto responsabili del funzionamento delle aziende. Infatti, i manager hanno la funzione/responsabilità di mantenere l'equilibrio delle aziende con riferimento ai bisogni di tutti i pazienti (e non solo quelli che afferiscono a specifiche unità organizzative o gruppi di professionisti), nonché l'equilibrio tra entrate disponibili e spese. Si è già sottolineato che lo sviluppo delle conoscenze ha determinato un'accelerazione nella pressione verso un aumento delle spese in un contesto nel quale le risorse 
pubbliche sono limitate e aumentano in misura meno rilevante rispetto al passato, almeno in Italia. A ciò si aggiunge che in un certo numero di casi, non importa se molti o in maggioranza, coloro che assumono le responsabilità manageriali di livello intermedio (direzione di unità organizzative, di dipartimenti, di distretti o a livello di vertice DG, DA, DS) non hanno conoscenze e competenze adeguate per affrontare i nuovi livelli di complessità e quindi agiscono in modo più accentuato sul "razionamento delle risorse" più che sulla razionalizzazione e innovazione gestionale, organizzativa, amministrativa, contabile.

Affrontare le nuove sfide con modelli culturali e con competenze manageriali del passato significa dare maggior peso ai vincoli rispetto alle opportunità derivanti dalla razionalizzazione-innovazione. In presenza di autonomia e responsabilità professionale per gli atti e i servizi erogati, i professionisti avvertono la logica dei vincoli o degli obiettivi di carattere economico come interferenza che mina la loro fiducia. A ciò si aggiunge il fatto che in un certo numero di casi chi assume ed esercita funzioni di tipo manageriale è ancora influenzato da un modello di tipo comando-controllo che si è sviluppato soprattutto con riferimento al mondo delle imprese private ma che non è adatto a governare professionisti dotati di autonomia. Un modello che appare inadeguato ed è messo in discussione dalle teorie e dalle esperienze riguardanti le imprese private e che a maggior ragione non si adatta a un sistema di tutela della salute ormai orientato alla personalizzazione dell'assistenza sia con riguardo alle condizioni di pazienti acuti sia alla presa in carico di persone con cronicità o disabilità multidimensionale.

Manager che non riescono a far percepire ai professionisti che le regole dell'efficiente funzionamento delle aziende sono finalizzate a migliorare il livello quali-quantitativo delle risposte ai bisogni di salute contribuiscono a ridurre il "patrimonio di fiducia" da parte dei professionisti. Oltre al burn-out legato alla professione (per esempio, chi opera nelle strutture di cure intensive o in condizioni di stress) si hanno anche forme di burn-out si tipo organizzativo collegato alla percezione che i vincoli imposti non consentano l'esercizio secondo deontologia professionale. Insieme alla fiducia viene eroso il patrimonio di tipo vocazionale e valoriale tipico delle professioni legate alle condizioni di salute delle persone. L'esercizio di funzioni manageriali in termini inadeguati e non innovativi contribuisce a ridurre il senso di appartenenza all'azienda da parte dei professionisti il cui sintomo è evidente nei casi sempre più numerosi di coloro che lasciano le aziende e gli ospedali pubblici appena ne hanno le condizioni giuridiche o che addirittura anticipano l'uscita prima del raggiungimento dei limiti di età. In un recente incontro informale ho sentito affermare da alcuni direttori generali di aziende di una Regione italiana che la loro maggiore difficoltà è quella di mantenere alta la motivazione dei professionisti, di far percepire un senso di appartenenza all'azienda e non solo alla propria unità organizzativa, di aiutarli a resistere in presenza di condizioni sempre più difficili su un piano delle pressioni derivanti da pazienti e loro associazioni, enti locali, richieste di produrre dati e documentazione, impossibilità di sostituire personale e altri vincoli. Parafrasando una battuta un po' cinica che riguarda le prestazioni professionali secondo cui occorre evitare situazioni in cui "l'operazione è riuscita ma il paziente è morto", si può dire che i manager devono evitare una situazione nella quale "l'azienda funziona bene, raggiunge le performance di tipo economico-finanziario, ma i professionisti abbandonano il campo e i pazienti trovano crescenti difficoltà ad avere risposte di salute". Purtroppo la catena fiducia $\rightarrow$ credibilità $\rightarrow$ comportamenti presenta rotture o segnali di significativa crisi anche nel rapporto tra manager delle aziende sanitario-ospedaliere e responsabili delle politiche di salute regionali e statali, più in generale della "sfera politica". In una società caratterizzata dalla volatilità del consenso politico i manager delle aziende pubbliche e private del settore salute si trovano a operare in un sistema di regole che sono obsolete, non coerenti con le nuove conoscenze, di incerta interpretazione, non di rado contraddittorie o lacunose. In questo contesto anche il patrimonio di fiducia dei manager sia riguardo all'adeguatezza delle proprie conoscenze e competenze sia riguardo alla possibilità di rispettare gli obiettivi a essi assegnati viene eroso. Il rischio è quello di avere manager che si appiattiscono su comportamenti di tipo esecutivo e burocratico finalizzati a minimizzare i rischi, non sono premiati o sono addirittura penalizzati da scelte innovative che sempre comportano rischi, abbandonano il campo e, come si suol dire, alzano bandiera bianca. Per fortuna del nostro Paese, di fronte a questi rischi di "selezione avversa" dei manager di aziende e di policy-makers condizionati da "logiche che perseguono il consenso di breve periodo", si hanno ancora molte persone che si impegnano e si mettono in gioco per cercare di mantenere un SSN basato su principi di universalità, solidarietà, equità. 
Se si condivide l'analisi che il "male oscuro" del SSN è quello della carenza di fiducia a vari livelli, occorre convenire che la terapia principale è quella di politiche, interventi, azioni idonee a immettere nel sistema dosi massicce di fiducia nei diversi punti che sono stati richiamati in questo editoriale. Il sostegno della ricerca nel settore delle life science, la promozione e l'incentivazione dell'innovazione tecnologica (farmaci, devices, terapie digitali ecc.), l'aumento delle risorse destinate al settore delle scienze della vita, la formazione di nuove professionalità, la semplificazione amministrativa, la razionalizzazione gestionale e organizzativa, l'adozione di nuovi modelli assistenziali (presa in carico delle persone con cronicità, organizzazione a rete ecc.) fanno parte della terapia, ma da soli non possono risolvere il problema. Il pilastro della terapia deve essere rappresentato da ricostituzione, potenziamento, consolidamento del "patrimonio di fiducia", che nel caso specifico parte dalla valorizzazione delle risorse che possono essere messe in campo dagli stessi pazienti o dalle persone che, tramite la prevenzione primaria, secondaria, terziaria, non contribuiscono ad aumentare la domanda di servizi e a incrementare la spesa pubblica o privata. Tutte le altre politiche possono considerarsi come terapie "coadiuvanti" della terapia principale. Si tratta peraltro di una terapia che riguarda non solo il settore di tutela della salute, ma che può aiutare ad affrontare la crisi delle democrazie rappresentative, tema introdotto all'inizio. Una terapia che richiede una riflessione profonda da parte di ogni persona, dei vari gruppi sociali, dei movimenti sociali e politici, dei rappresentanti delle istituzioni pubbliche e private sul rapporto diritti/doveri. La società ha da troppi decenni posto vari livelli di attenzione soprattutto sui diritti, dimenticando troppo spesso che di fronte a ogni diritto sta un dovere della stessa persona o di altre persone che devono avere comportamenti idonei a garantire il diritto. I pazienti hanno il diritto costituzionale e derivante dalla legge n. 833/78 di istituzione del SSN di ricevere risposte ai propri bisogni di salute nell'ambito dei livelli essenziali di assistenza definiti dallo Stato e dalle Regioni, ma hanno il dovere di assumere comportamenti responsabili nei confronti dell'uso dei servizi. I professionisti hanno il diritto di svolgere la propria attività con autonomia, ma hanno il dovere di rispondere ai bisogni reali dei pazienti e di contribuire al buon funzionamento delle aziende. I manager hanno il diritto di rivendicare regole chiare e non contraddittorie e di avere a disposizione risorse coerenti con i fabbisogni, ma hanno il dovere di continuare a migliorare la propria professionalità e di innovare. I politici hanno il diritto di adottare scelte pensando anche all'ottenimento del consenso, ma hanno il dovere di acquisire conoscenze dirette o indirette necessarie per definire priorità di salute e di avere una visione di lungo periodo. Infine, va sottolineato che la fiducia è un bene intangibile che aumenta man mano con il suo utilizzo. Chi assume atteggiamenti di fiducia contribuisce a stimolare fiducia, anche se purtroppo molte volte ciò non accade e quindi ognuno deve essere disposto a correre rischi che sono tipici delle relazioni e in definitiva della vita. 